\title{
Analysis of the characteristics of terminal ileal ulcers: prognosis and clinical significance
}

\section{Liping Yang}

Zhejiang University School of Medicine First Affiliated Hospital

\section{Zhongchen Zhang}

Zhejiang University School of Medicine First Affiliated Hospital

\section{Xinxin Zhou}

Zhejiang University School of Medicine First Affiliated Hospital

\section{Chunxiao Chen ( $\nabla 1516030 @ z j u . e d u . c n)$}

Zhejiang University School of Medicine First Affiliated Hospital

\section{Research article}

Keywords: terminal ileal ulcer; prognosis

Posted Date: July 24th, 2019

DOI: https://doi.org/10.21203/rs.2.11885/v1

License: (c) (1) This work is licensed under a Creative Commons Attribution 4.0 International License. Read Full License 


\section{Abstract}

Background Although terminal ileal ulcers can be observed occasionally in colonoscopy examination, the prognosis, clinical significance and treatment guidelines are unclear. Methods All patients diagnosed with terminal ileal ulcers from March 2014 to March 2018 were enrolled. We extracted data of gender, age, clinical symptoms, number of terminal ileal ulcers, pathological outcome, treatment programs. Results Of 42 individuals, one patient diagnosed with tuberculosis and received anti-tuberculosis treatment. The other 41 individuals chose to follow up or 5-ASA plus probiotics treatment. Logistic regression analysis showed no significant difference in treatment selection and gender $(P=0.848)$, age $(P=0.481)$, ulcers $(p=0.116)$, symptoms $(P=0.326)$. All individuals had good prognosis. Conclusion Individuals with terminal ileal ulcers have a good prognosis regardless of follow-up or treatment.

\section{Introduction}

The terminal ileum refers to ileum approximately $30 \mathrm{~cm}$ in length from the ileocecal valve to anal side of the ileum. This location is a predilection site for small bowel disease. The long-term clinical findings indicate that the onset of this site is occult and the patient's clinical symptoms and signs are often not obvious on the early stage, which makes early diagnosis more difficult. Crohn's disease[1], intestinal tuberculosis[2], and small intestinal lymphoma[3] all occur in terminal ileum, and early diagnosis is difficult. The characteristics of these diseases can be manifested as congestion, edema, ulcers and even bleeding in terminal ileum. In recent years, with the continuous improvement of medical technology, colonoscopy has been widely popularized. Combining colonoscopy diagnosis technology with histopathological results greatly improves the diagnostic accuracy rate of terminal ileum lesions[4]. In addition to these digestive diseases, the detection rate of simple ileal ulcer lesions is also increasing while the disease itself is not specific.

In our study, we aimed to collect patients diagnosed as terminal ileal ulcers by colonoscopy and to evaluate the diagnostic value and diagnostic accuracy of electronic colonoscopy and histopathological examination for terminal ileal ulcers. In addition, through analyzing characteristics of various types of terminal ileal ulcers, we try to contribute to the understanding of the diagnosis and differential diagnosis of terminal ileal ulcers.

\section{Methods And Materials}

\section{Patients and diagnostic criteria}

We screened about 40,000 individuals who underwent colonoscopy in our hospital from March 2014 to March 2018. During this period, 42 individuals diagnosed as terminal ileal ulcers without colorectal lesions and the detection rate was $0.1 \%$ (Figure 1). This study was approved by our Institutional Review Board. Informed consent was obtained and privacy was respected. The experimental protocol was approved by the Ethics Committee of the First Affiliated Hospital, College of Medicine, Zhejiang University 
(2018-965). In addition, all procedures were performed in accordance with the relevant guidelines and regulations. We had access to the study data.

The inclusion criteria were as follows: (1) age > 18 years old; (2) no history of other gastrointestinal ulcers; (3) no long-term medication history includes corticosteroids, immunosuppressants or NSAIDs; (4) no other chronic disease history including tumors, tuberculosis, autoimmune diseases, etc.

\section{Data extraction}

We extracted data of gender, age, clinical symptoms, number of terminal ileal ulcers, pathological outcome, treatment programs. The symptoms of 42 individuals were mainly abdominal pain, diarrhea, constipation, dyspepsia, and physical examination.

\section{Statistical analysis}

Continuous variables were expressed as a mean \pm standard deviation. Student's t-test was used to compare the difference of age between different subgroups. Chi-square test is used to compare categorical variables, such as gender, ulcer. Logistic regression is used to compare the relationship between drug selection and multiple variables. The statistical significance was defined as $P \leq 0.05$. We used SPSS 21.0 (IBM, Chicago, IL, USA) to perform the statistical analysis.

\section{Results}

The baseline characteristics were shown in Table 1. Among the 42 individuals selected, clinical symptoms were divided into five categories (33.3\% was abdominal pain, $28.6 \%$ was diarrhea, $14.3 \%$ was constipation and dyspepsia, $9.5 \%$ was physical examination, $11.9 \%$ was others). Number of terminal ileal ulcers under the colonoscope, $59.5 \%$ was single and $40.5 \%$ was multiple. One patient diagnosed with tuberculosis, because pathological results showed positive acid-fast staining and positive hematological examination of T-SPOT. Then administration of anti-tuberculosis treatment was given. The pathological results of the remaining 41 individuals all showed chronic inflammation of the mucosa. 6 of 41 individuals conducted subsequent examination of small bowel capsule endoscopy (SBCE). The results showed terminal ileal ulcers without other ulcers of gastrointestinal tract. Treatment options included 5ASA plus probiotics and follow-up. After at least half a year of follow-up, there was no progression in outpatients, and the ulcer was healed in 5 patients who performed colonoscopy.

Different treatment options seem to have no effect on the patient's prognosis. There was no statistical difference in gender $(P=0.702)$, age $(P=0.696)$, ulcer $(P=0.144)$ between the two treatments.

Logistic regression analysis also showed that there was no significant difference in gender $(P=0.848)$, age $(P=0.481)$, ulcers $(p=0.116)$, symptoms $(P=0.326)$.

\section{Discussion}


In our study, our findings indicated that the asymptomatic individuals with terminal ileal ulcers had a favorable clinical course without critical events. Chang HS et al reported similar results about terminal ileal ulcerations in asymptomatic individuals[5]. The cause of ulcerative lesions at terminal ileal is not completely clear. It may be related to bacterial colonization at terminal ileal to form an infection and induce an immune response, which ultimately causes damage to the mucosa. Some scholars believe that it is caused by autoimmune dysfunction, causing paralysis of blood vessels and muscles in the intestinal wall, causing impaired cell nutrition in the intestinal wall and decreased resistance of the intestinal mucosa, resulting in intestinal mucosa susceptible to intestinal juice erosion and ulceration[6]. While some scholars believe that it is caused by Campylobacter infection[6].

Small ulcerations in the terminal ileum may be one of the earliest manifestations of serious diseases, such as Crohn's disease. Some studies reported that patients with aphthous-type Crohn's disease may later develop typical Crohn's disease[7, 8]. There was also researcher who hold the opposite view. Courville EL et al has reported that Crohn's disease is unlikely to develop in asymptomatic individuals with isolated ileitis[9]. In our study, we also did not find the development of Crohn's disease. Moreover, long-term follow-up studies and prospective multicenter study are needed to assess this hypothesis accurately, because this progression may take place over after a period of time.

There were a few limitations in our study. Firstly, limited number of individuals enrolled. In our center, only 42 individuals $(0.1 \%)$ had terminal ileal ulcers. Due to differences in individuals' compliance, some individuals had poor bowel preparation, which would affect observation and miss lesions. In addition, some very small ulcers may be replaced by erosion under the judgment of some doctors. These limitations may have led to an underestimation of the actual prevalence of terminal ileal ulcers in this study. Second, Although the included individuals were diagnosed with isolated terminal ileal ulcers by colonoscopy, only a few individuals underwent capsule endoscopy. Therefore, we could not exclude the possibility that included individuals with ulcerations in the small bowel. 6 of 42 individuals performed capsule endoscopy and found other intestinal ulcers in our study. Third, this study did not involve the study of etiology, pathogenesis and risk factors. Moreover, the results of this study also did not represent other countries, especially the West. Finally, although we have followed the individuals for a certain period of time, we still don't know the longer-time evolution of the lesions. Therefore, we can't make any definite recommendations on how to manage these lesions.

\section{Conclusions}

In conclusion, we have shown that individuals with terminal ileal ulcers have a good prognosis regardless of follow-up or treatment. Even if these lesions exist, it is not clear whether there is a connection with the symptoms of the patients. These findings provided a suggestion that we can choose to follow up for isolated terminal ileal ulcers. However, we still recommend that patients undergo capsule endoscopy if economic conditions permit. Moreover, we need more prospective multicenter long-term follow-up studies to confirm the perspective of this study. 


\section{Declarations}

Ethics approval and consent to participate: The experimental protocol was approved by the Ethics Committee of the First Affiliated Hospital, College of Medicine, Zhejiang University (2018-965)

Consent for publication: Not applicable

Availability of data and material: The datasets used and/or analysed during the current study are available from the corresponding author on reasonable request.

Competing interests: The authors declare that they have no competing interests

Funding: This article was Supported by National Natural Science Foundation of China (81600414) and the Natural Science Foundation of Zhejiang Province (LQ16H030001).

Authors' contributions: Liping Yang designed research; Liping Yang collected material and clinical data from patients; zhongchen Zhang and Xinxin Zhou performed the assays; Liping Yang wrote the paper; Chunxiao Chen revised the paper.

Acknowledgements: Not applicable

Authors' information (optional): Not applicable

\section{References}

1. Baumgart DC, Sandborn WJ: Crohn's disease. Lancet (London, England) 2012, 380(9853):1590-1605.

2. Makharia GK, Srivastava S, Das P, Goswami P, Singh U, Tripathi M, Deo V, Aggarwal A, Tiwari RP, Sreenivas $\vee$ et al: Clinical, endoscopic, and histological differentiations between Crohn's disease and intestinal tuberculosis. The American journal of gastroenterology 2010, 105(3):642-651.

3. Catassi C, Bearzi I, Holmes GK: Association of celiac disease and intestinal lymphomas and other cancers. Gastroenterology 2005, 128(4 Suppl 1):S79-86.

4. Jeong SH, Lee KJ, Kim YB, Kwon HC, Sin SJ, Chung JY: Diagnostic value of terminal ileum intubation during colonoscopy. Journal of gastroenterology and hepatology 2008, 23(1):51-55.

5. Chang HS, Lee D, Kim JC, Song HK, Lee HJ, Chung EJ, Kim TH, Park HW, Byeon JS, Yang SK et al: Isolated terminal ileal ulcerations in asymptomatic individuals: natural course and clinical significance. Gastrointestinal endoscopy 2010, 72(6):1226-1232.

6. Tang S, Yuan H, Qin L: Differential diagnostic values of double-balloon enteroscopy in Crohn's disease and intestinal tuberculosis. World Chinese Journal of Digestology 2007, 15(19):2159-2161.

7. Hizawa K, lida M, Kohrogi N, Kuroki F, Yao T, Sakamoto K, Fujishima M: Crohn disease: early recognition and progress of aphthous lesions. Radiology 1994, 190(2):451-454.

8. Matsumoto T, lida M, Nakamura S, Hizawa K, Yao T, Fujishima M: Crohn's disease of aphthous type: serial changes in intestinal lesions. The British journal of radiology 2000, 73(874):1046-1051. 
9. Courville EL, Siegel CA, Vay T, Wilcox AR, Suriawinata AA, Srivastava A: Isolated asymptomatic ileitis does not progress to overt Crohn disease on long-term follow-up despite features of chronicity in ileal biopsies. The American journal of surgical pathology 2009, 33(9):1341-1347.

\section{Table}

\begin{tabular}{|c|c|c|}
\hline Parameter & 5-ASA + probiotics & Follow-up \\
\hline \multicolumn{3}{|l|}{ Gender } \\
\hline Female & 6 & 12 \\
\hline Male & 9 & 14 \\
\hline Age $\rrbracket$ mean $\pm S D \otimes$ & $43.5 \pm 15.5$ & $43.6 \pm 15.5$ \\
\hline \multicolumn{3}{|l|}{ Ulcer } \\
\hline Single & 11 & 13 \\
\hline multiple & 4 & 13 \\
\hline \multicolumn{3}{|l|}{ Symptoms } \\
\hline abdominal pain & 5 & 9 \\
\hline diarrhea & 5 & 7 \\
\hline constipation and dyspepsia & 2 & 4 \\
\hline physical examination & 0 & 4 \\
\hline others & 3 & 2 \\
\hline
\end{tabular}

\section{Figures}

\section{Figure 1}

olonoscopic images showed aphthous or small ulcerations in patients with terminal ileal ulcerations.

\section{Supplementary Files}

This is a list of supplementary files associated with this preprint. Click to download.

- InstitutionalReviewBoardApproval.pdf 